Sybille Krämer · Sibylle Schmidt (Hg.)

Zeugen in der Kunst 

Sybille Krämer · Sibylle Schmidt Hg.

\section{Zeugen in der Kunst}

Wilhelm Fink 
Umschlagabbildung:

Dadang Christanto: Litsus (2004), Performance am 31. Mai 2015 am „4A Centre for

Contemporary Asian Art“ in Sydney, im Rahmen von „MASS GROUP INCIDENT:

48HR Incident“. Foto: Pedro de Almeida. Mit freundlicher Genehmigung des Künstlers, sowie Marita Smith von „Gallerysmith“ in Melbourne und dem „4A Centre for

Contemporary Asian Art“ in Sydney.

Gedruckt mit Unterstützung der Deutschen Forschungsgemeinschaft.

Bibliografische Information der Deutschen Nationalbibliothek

Die Deutsche Nationalbibliothek verzeichnet diese Publikation in der Deutschen

Nationalbibliografie; detaillierte bibliografische Daten sind im Internet über http://dnb.d-nb.de abrufbar.

Alle Rechte, auch die des auszugsweisen Nachdrucks, der fotomechanischen Wiedergabe und der Übersetzung, vorbehalten. Dies betrifft auch die Vervielfältigung und Übertragung einzelner Textabschnitte, Zeichnungen oder Bilder durch alle Verfahren wie Speicherung und Übertragung auf Papier, Transparente, Filme, Bänder, Platten und andere Medien, soweit es nicht $\$ \$ 53$ und 54 UrhG ausdrücklich gestatten.

(C) 2016 Wilhelm Fink, Paderborn

(Wilhelm Fink GmbH \& Co. Verlags-KG, Jühenplatz 1, D-33098 Paderborn)

Internet: www.fink.de

Einbandgestaltung: Evelyn Ziegler, München

Printed in Germany

Herstellung: Ferdinand Schöningh GmbH \& Co. KG, Paderborn

ISBN 978-3-7705-6020-2 
Aurélia Kalisky

\title{
DiE ERZEUGUNG DER WAHRHEIT \\ ZWISCHEN KUNST UND ZEUGENSCHAFT. ÜBER EIN IN AUSCHWITZ GESCHRIEBENES LITERARISCHES MANIFEST
}

\begin{abstract}
Am 3. Januar 1945, kurz vor der Befreiung des Konzentrationslagers Auschwitz durch die Rote Armee, schrieb ein Gefangener namens Abraham Levite einen Text, der als Einleitung eines Sammelbands mit dem Titel Auschwitz dienen sollte. Ziel dieser Anthologie war es, „die Gedichte, Beschreibungen und Eindrücke des [von den im Konzentrationslager Gefangenen, A.K.] Gesehenen und Erlebten“" zu sammeln, „um ihr Leiden wiederzugeben, ihre Verbitterung auszudrücken und, in gewisser Weise, ihre Leben zu rechtfertigen ". ${ }^{1}$ Die innerhalb des Lagers geschriebenen Texte sollten mithilfe des Widerstands heimlich hinausgebracht werden und dazu dienen, die Welt über die Geschehnisse in Auschwitz zu informieren. Aber der Vormarsch der Roten Armee und die darauffolgende Evakuierung des Lagers verhinderten die Weitergabe der Texte an den polnischen Widerstand, sodass alle im Geheimen verfassten Manuskripte gemeinsam mit ihren Autoren untergingen. Abraham Levite war wahrscheinlich der einzige Überlebende von ihnen und seine Einleitung der einzige Text, der aus dieser Anthologie verblieb und übermittelt wurde. ${ }^{2}$ Er beinhaltet folgende Zeilen:
\end{abstract}

1 Mit diesen Worten wurde die „Einleitung des Sammelband-Projekts Auschwitz“ in der Zeitschrift des Jiddischen Wissenschaftlichen Instituts in New York (Yidisher Visnshaftlekher Institut oder YIVO) präsentiert, als sie erstmals in ihrer jiddischen Originalfassung im Frühjahr 1946 unter dem Titel „Dos samlbukh Oyshvits“ veröffentlicht wurde (YIVO Bleter, Nr. 27, S. 194-197). Die Identität des Autors dieser knappen, einleitenden Bemerkung, in Rouen, Frankreich, am 14. Juni 1945 fertiggestellt, bleibt unklar. Wahrscheinlich handelt es sich jedoch um den Rabbiner Morris Dembovitz, dem Abraham Levite seinen Text in Stuttgart nach seiner Befreiung aus Auschwitz anvertraut hatte.

2 Laut eines Vermerks der YIVO-Bleter-Redaktion gab vermutlich Morris Dembovitz den Text von Abraham Levite über zwei Mitglieder des Jüdischen Theologischen Seminars von New York an das YIVO weiter. Levite hat viel später eigenständig seinen Text in Israel auf Jiddisch und Hebräisch in einem so genannten Memorbuch bzw. Jizkor-Buch unter dem Titel Sefer sikaron kehilat Bresiv (Brzozow) (http://yizkor.nypl.org/index.php?id=1027) veröffentlicht. In Frankreich wird der Text von Levite in einer Gesamtedition der Handschriften der Sonderkommandos von Auschwitz einmal als anonymer, in Auschwitz I aufgefundener Text präsentiert, gleichzeitig aber im Inhaltsverzeichnis einem Mitglied des Sonderkommandos aus Auschwitz II-Birkenau zugeschrieben, wo er unter dem Titel „Die Anthologie Auschwitz. Text von einem Mitglied des Sonderkommandos, 3. Januar 1945" aufgeführt wird. Siehe Des voix sous la cendre. Manuscrits des Sonderkommandos d'Auschwitz-Birkenau, Paris: Mémorial 
„Ich las einmal über Menschen, die zum Nordpol wollten und deren Schiff im Eis stecken blieb; ihre S.O.S.-Rufe blieben ohne Antwort. Die Nahrungsmittel gingen ihnen aus, der Frost hatte sie fest im Griff, und sie warteten abgeschnitten von der Welt, erfroren und verhungert auf den Tod. Trotzdem ließen diese Menschen den Bleistift nicht aus ihren froststarren Händen. Sie machten weiter Notizen in ihre Tagebücher, vor ihren Augen schwebte die Ewigkeit.

Wie gerührt war ich davon: dass Menschen unter solchen tragischen Bedingungen, die das Leben so unbarmherzig von sich gestoßen und auf die der Tod schon seine Klauen gelegt hatte, dass diese Menschen sich so über ihr Schicksal erhoben und weiter ihrer Pflicht für die Ewigkeit nachkamen.

Wir alle, die wir hier in der polaren, eiskalten Gleichgültigkeit der Völker sterben, von Welt und Leben vergessen, haben doch das Bedürfnis, der Ewigkeit etwas zu hinterlassen, wenn schon keine vollständigen Dokumente, so doch wenigstens Bröckchen davon: wie wir, lebendige Tote, erinnerten und fühlten, dachten und redeten. Auf den Gräbern, in denen wir lebendig begraben liegen, tanzt die Welt ihren Teufelstanz und übertrampelt mit den Füßen unser Stöhnen und unsere Hilferufe. Wenn wir dann schon erstickt sind, wird man sich daran machen, uns auszugraben. Uns wird es dann nicht mehr geben, nur unsere über die sieben Meere zerstreute Asche, dann wird es jeder kultivierte und anständige Mensch für seine Schuldigkeit ansehen, uns zu bedauern und Nachrufe auf uns zu halten. Wenn unsere Schatten auf Leinwänden und Kanzeln erscheinen, werden mitleidige Damen sich mit ihren parfümierten Taschentüchern die Augen wischen und uns beklagen: ach, ihr Unglücklichen!

Wir wissen, wir kommen hier nicht lebendig heraus. An das Tor dieser Hölle hat der Teufel eigenhändig geschrieben: ,Lasst, die ihr eingeht, alle Hoffnung fahren!' Wir wollen Zeugnis ablegen, das soll unser Schma Jisrael sein vor den kommenden Generationen. Das soll das Bekenntnis einer tragischen Generation sein, einer Generation, die ihrer Aufgabe nicht gewachsen war, deren rachitische Beine unter der schweren Last des Martyriums zusammengebrochen sind, die die Zeit uns auferlegt hatte.

Und deshalb: Es geht uns hier nicht um Fakten und Ziffern, nicht darum, trockene Dokumente zu sammeln - das wird auch ohne uns getan werden. Auch ohne unsere Mithilfe wird die Geschichte von Auschwitz zusammengestellt werden können. Wie in Auschwitz gestorben wurde, werden Bilder, Zeugnisse, Dokumente erzählen. Wir aber wollen hier ein Bild davon schaffen, wie in Auschwitz ,gelebt' wurde. Wie ein normaler, durchschnittlicher Arbeitstag im

de la Shoah/Calmann-Lévy, 2005, S. 175-181 und S. 441. Ein wenig irreleitend ist auch die jüngste Veröffentlichung des Textes in Russland, wo er in einem Buch von Pavel Polian über die Handschriften der Sonderkommandos veröffentlicht und kommentiert wurde: Das Jüdische "Sonderkommando" in Auschwitz-Birkenau und seine Chronisten, Moskau/Rostow am Don 2013. Die genauen Bedingungen der Überlieferung des Textes und dessen Geschichte bedürfen nach wie vor einer eingehenderen Untersuchung. Zur komplexen Veröffentlichungs- und Rezeptionsgeschichte des Textes, u. a. in Israel, und ihrer kulturgeschichtlichen Implikationen sei an dieser Stelle auf meinen Artikel verwiesen: „Les archives de la douleur en yiddish. L'odyssée d'un texte, du camp d'Auschwitz à la Knesset“, in: Judith Lindenberg (Hg.), Écritures de la destruction, Paris, im Druck. 
Lager aussah. Ein Tag, der ein Durcheinander von Leben und Tod war, von Angst und Hoffnung, von Resignation und Lebenswillen. Ein Tag, in dem die eine Minute nicht weiß, was die nächste mitbringen wird. Wie man gräbt und mit dem Beil Stücke des eigenen Lebens abhackt, blutige Stücke, Jugendjahre, und wirft sie keuchend auf die Lore der Zeit, die so jammervoll kreischt, so bitter schwer läuft auf den eisernen Gleisen der Lagerroutine. Und abends wird aus der Lore ein zu Tode Gequälter in den tiefen Abgrund gekippt. Ach, wer wird einen so blutigen Tag zusammen mit seinem Schatten, einer mit Ängsten begossenen Nacht, herausholen und es der Welt zeigen?

[...] Wir müssen selbst erzählen was uns betrifft. Wir sind uns bewusst, dass es uns an Kraft fehlt, alles aufzuschreiben und etwas zu schaffen, was unsere Tragödie ausdrücken und abspiegeln könnte. Aber - was wir schreiben, darf ohnehin nicht auf der literarischen Waage gewogen werden. Es muss als Dokument betrachtet werden, und also ist nicht auf den künstlerischen Wert der Sache zu achten, sondern Zeit und Ort müssen berücksichtigt werden. Und die Zeit ist: kurz vor dem Tod; und der Ort ist: auf dem Schafott. Nur vom Artisten auf der Bühne verlangt man, dass er wenig schreit und dass er, wenn er stöhnt, es nach allen Regeln der Kunst tut. Denn ihm tut ja nichts weh. Das betroffene Opfer aber wird letzten Endes niemand dafür kritisieren, dass es zu laut stöhnt oder zu leise weint.

Und wir haben etwas zu sagen, auch wenn wir, literarisch genommen, Stotterer sind. Wir werden erzählen, so gut wir können, in unserer Sprache. Auch ganz Stumme können nicht schweigen, wenn es ihnen weh tut, sie reden dann, aber in ihrer Sprache: in Stummensprache. Schweigen, das tun nur die Bonzes. Die machen ein geheimnisvolles Gesicht, als hätten sie wer weiß was zu sagen. Aber erst dort in der Welt der Wahrheit, wo Pose und Getue nichts mehr gelten, kommen sie mit dem Geheimnis ihres Lebens heraus: einem Brötchen mit Butter.

\section{[...]}

Also, Kameraden, schreibt, zeichnet kurz und scharf, kurz wie die Tage, die uns noch zum Leben bleiben, und scharf wie die Messer, die auf unsere Herzen zielen. Sollen ein paar Blättchen fürs YIVO bleiben, fürs jüdische SchmerzArchiv, sollen es unsere am Leben gebliebenen freien Brüder lesen; und vielleicht werden sie davon auch etwas lernen.

\section{Und wir beten zum Schicksal}

Es sei der Wille bei Dir

Nicht hört Er die Stimme des Weinens

So tu uns wenigstens das - dass du unsre Tränen bei Dir sein lässt

Verbirg die Tränenblättchen im Behältnis des Seins

lass sie kommen in richtige Hände

und finden ihren Tikkun."

3 Ich zitiere hier ungefähr die Hälfte des Textes in einer unveröffentlichten Übersetzung aus dem Jiddischen von Almut Seiffert. 
Mit seinem Text, der unter den widrigsten und grauenhaftesten Umständen entstand, formuliert Abraham Levite mit einer erstaunlichen Klarsicht die Erkenntnis einer Art anthropologischer Wende in der Geschichte der Gewalt im 20. Jahrhundert. Diese Wende erscheint als maßgebend für die neuen Formen von Zeugenschaft, die im Zuge der historischen Katastrophen der Gegenwart von einem „Zeitalter des/der Zeugen" sprechen lassen. ${ }^{4}$ Indem Levites Text die entscheidenden Fragen stellt, die aus der Konfrontation zwischen Kunst und dieser neuen Form der Gewalt entstehen, geht er noch weit über diese Erkenntnis hinaus. Sein Text kann dementsprechend als Manifest für eine Neubegründung der Kunst - in diesem Fall vor allem der Literatur - im Lichte, oder besser gesagt, im Schatten der genozidalen Katastrophe interpretiert werden. Dadurch, dass diese neue Kunst der Zeugenschaft, welche hier im Moment der tiefsten Verzweiflung heraufbeschworen wird, versucht, oft völlig entgegengesetzte Intentionen zu vereinen, weist sie durchweg paradoxe Züge auf. Eigentlich ist aber diese, scheinbar in sich widersprüchliche Geste als eine multidimensionale zu interpretieren. Die Poetik eines literarischen Zeugnisses, wie sie im Moment der größten Gefahr definiert wird, beinhaltet in der Tat mehrere Dimensionen des Zeugnis-Ablegens: Das Attestieren einer Realität durch ein Dokument schließt hier keineswegs das Vermitteln einer subjektiven und existentiellen Wahrheit durch das literarische Schreiben aus. Gleichzeitig soll die persönliche Geschichte des zum Schriftsteller gewordenen Zeugen, aber vor allem die kollektive Geschichte der Opfer, für die kommenden Generationen sowohl eine Lektion darstellen als auch ein Archiv bilden. Und genauso wie die hier definierte Kunst des Zeugnisses ein Dokument zum „Schmerz-Archiv“ werden lässt, soll auch umgekehrt die Kunst selbst durch das Zeugnis einer ungeheuren menschlichen Erfahrung der Unmenschlichkeit sich ändern und sich davor bewahren, selbst zu einem „Dokument der Barbarei“" zu werden. So wird im Text von Abraham Levite eine Poetik definiert, die sich zwischen Kunst und Zeugenschaft platziert, um nicht nur die Wahrheit der Ereignisse zu bezeugen, sondern auch seine zu übermittelnde Wahrheit des Erlebten zu erzeugen.

4 Siehe Annette Wieviorka: L'Ère du témoin, Paris 1998; Shoshana Felman: „Im Zeitalter der Zeugenschaft: Claude Lanzmanns Shoab“, in: Ulrich Baer (Hg.): ,Niemand zeugt für den Zeugen'. Erinnerungskultur nach der Shoah, Frankfurt am Main 2000, S. 173-192, erstmals 1990 in den USA und in Frankreich veröffentlicht; Claude Burgelin: „Le temps des témoins“, in: Cabiers de la Villa Gillet, Nr. 3, Nov. 1995, S. 79-89.

5 Walter Benjamin: „Über den Begriff der Geschichte“, in: Gesammelte Werke, Bd. I/2, Frankfurt am Main 1991, S. 690-708. 


\section{Wandel der Gewalt und Angriff auf das Zeugnis}

Als er seine Einleitung heimlich verfasst, hat Abraham Levite als polnischer Jude bereits den gesamten Prozess der Vernichtung des europäischen Judentums erlebt. ${ }^{6}$ Die Shoah ist keineswegs das erste historische Ereignis, das jene neue Form der Gewalt impliziert, die vom Nationalstaat ausgeübt wird und für sich in Anspruch nimmt, die Zugehörigkeit zur Menschheit zu bestimmen. Da sie aber als Grundlage für die rechtliche Festlegung des Konzepts des, Genozids ' diente, ist sie dennoch gewissermaßen dessen bedeutendster Musterfall. ${ }^{7}$ Mit der Schaffung von Kategorien wie ,Verbrechen gegen die Menschheit' (1915), ,Verbrechen gegen die Menschlichkeit' (1945-46) und ,Genozid' (oder, Völkermord', 1948), die der strafrechtlichen Benennung von Verbrechen dienen sollten, die in vielen Fällen gerade im Namen des Rechts begangen worden waren, ${ }^{8}$ hat sich die menschliche Justiz in ihrer Formbarkeit gewissermaßen selbst verkehrt. Die Verbrechen werden vom Staat selbst, kraft der gesetzlich anerkannten Gewalt des Rechts begangen. In der Genozidforschung ist aus diesem Grunde vom „verbrecherischen Staat “9 die Rede. Aber die juridische Perspektive erlaubt nur eine teilweise und bedingte Erhellung der radikalen Neuartigkeit dieser Form von Gewalt, die auch aus anthropologischer, politikwissenschaftlicher sowie aus philosophischer Perspektive zu betrachten ist, handelt es sich doch um eine Gewalt, die einige menschliche Existenzformen schlichtweg aus der Menschheit ausschließt und dabei selbst Handlungen zum Vorschein bringt, welche die Definition dessen, was ,menschlich' ist, unterminieren.

Abraham Levite beschreibt dies so treffend wie kein anderer: Während die Polarreisenden vom „Leben so unbarmherzig von sich gestoßen“ werden, sind die Opfer von Auschwitz nicht nur vom „Leben“, sondern von der „Welt [...] vergessen“. Möglich wird dieses Vergessen durch das, was Levite als „polare[...], eiskalte[...]

6 Im Jizkor-Buch von Brzozow kann man Genaueres über seine Erfahrungen während des Krieges nachlesen: Nach dem Einmarsch der deutschen Truppen in seinem galizischen Schtetl versuchte Levite zuerst in die Sowjetunion zu fliehen, kehrte aber in seine Heimatstadt zurück, wo er die Deportation in das KZ Plaszow und die Hinrichtung der meisten Mitglieder seiner Familie wegen ,Arbeitsunfähigkeit' miterlebte. 1943 wurde er nach Auschwitz deportiert, überlebte jedoch das Lager und auch den Todesmarsch nach Zwickau über Gross-Rosen und Buchenwald.

7 Hier muss daran erinnert werden, dass der Begründer des Genozid-Begriffs, Rafael Lemkin, sich auch auf den Genozid an den Armeniern sowie an den Herero und Nama bezieht. Für eine historische und kritische Annäherung an die Entstehung des Konzepts siehe u.a.: Anson Rabinbach: Begriffe aus dem Kalten Krieg. Totalitarismus, Antifaschismus, Genozid, Göttingen: Wallstein, 2009; Dirk A. Moses: „The Holocaust and Genocide“, in: Dan Stone (Hg.): The Historiography of the Holocaust, New York 2004, S. 533-555; David Kazajian: „Re-flexion. Genocide in Ruins“, in: Discourse, Bd. 33, Nr. 3, Johannesburg, 2011, S. 367-389.

8 Siehe hierzu die Analysen Giorgio Agambens in: Mittel ohne Zweck. Noten zur Politik, Zürich/Berlin 2001 und in: Homo Sacer. Die souveräne Macht und das nackte Leben, Frankfurt am Main 2002.

9 Yves Ternon: L'État criminel. Les génocides au XXe siècle, Paris 1995. 
Gleichgültigkeit der Völker" bezeichnet. Der Philosoph Philippe Bouchereau war es, der erstmals das Neuartige an dieser „eiskalten Gleichgültigkeit“ auf eine begriffliche Ebene brachte, indem er von der "Nicht-Zugehörigkeit" („désappartenance“) zur Menschheit sprach, die durch den „Völkermord-Menschheitsmord“ ausgeführt wird und die innerhalb der Rechtskategorie,Verbrechen gegen die Menschlichkeit einen Sonderfall darstellt. ${ }^{10}$ Verfolgung und Vernichtung werden nämlich im Fall des Genozids nicht auf die physische Ausrottung der Gruppe beschränkt, sondern zielen ebenso auf die Auslöschung ihrer Erinnerung im ,gereinigten' kollektiven Gedächtnis ab. Leugnungsstrategien werden durch eine politische Organisierung des Staatsgeheimnisses und der Lüge zu einem wesentlichen Bestandteil der Planung und Ausführung des Verbrechens, oft ergänzt durch die materielle Vernichtung jeglicher Beweise der kriminellen Intention und nachträglich durch manipuliertes und instrumentalisiertes Wissen weitergeführt. In einer Zeit, in der geschichtswissenschaftliche Interpretationen historischer Ereignisse sowie deren Verrechtlichung zentral für die Etablierung der öffentlichen Wahrheit sind, ist die Erkenntnis der Negation als wesentlicher Bestandteil von Verbrechen gegen die Menschheit entscheidend, insofern wir es oft mit Verbrechen zu tun haben, bei denen es gerade durch die Leugnung der historischen Fakten an Evidenz und an Beweisen im juridischen sowie im historiografischen Sinne mangelt.

Dieses Phänomen führte Shoshana Felman bekanntlich dazu, vom Genozid als Angriff auf das für die moderne Wissensordnung grundlegende „westliche Gesetz der Evidenz" ${ }^{\text {"11 }}$ zu sprechen. Diesem liegen sowohl das Verfahren der juridischen Ermittlung, das zum Vorlegen von Beweisen vor Gericht führen soll, als auch der Prozess der geschichtswissenschaftlichen Untersuchung von vergangenen Ereignissen, der in der Erklärung von Fakten und somit deren Sinngebung und Interpretation münden soll, zugrunde. Zeugenschaft in Form von Augenzeugenberichten ist hier als imperfektes, dennoch oft unumgängliches Beweismittel von zentraler Bedeutung. Sie ist unersetzbar, zugleich strengstens reglementiert und kontrolliert, sei es im Recht oder in der Geschichtswissenschaft, sodass ihre Autorität immer

10 Philippe Bouchereau: „La désappartenance. Penser et méditer le génocide“, in: L'Intranquille, Nr. 4-5, Paris 1999, S. 165-211, ebenso „Le génocide est sans raison“, in: L'Intranquille, Nr. 6-7, Paris 2001, S. 279-297. Bouchereau unterscheidet zwischen Krieg, Ethnozid und Genozid einerseits und zwischen dem Wesen von Völkermord und totalitärer Gewalt andererseits. Während bei den Verbrechen gegen die Menschlichkeit die Menschlichkeit des Anderen ausgehend von der Reduktion seiner Einzigartigkeit auf ein Anders-Sein negiert und dadurch eine Negierung der Existenz einer einheitlichen Menschheit impliziert wird, ist der Genozid die Verneinung eines universalen Begriffs von Menschheit und damit die Verneinung der Menschheit an sich. Ausgehend von dieser Sichtweise schlägt Bouchereau vor, die Absicht, eine Gruppe als solche, aber ohne Grund, auszurotten, mit „Völkermord-Menschheitsmord“ (oder „Genozid-Humanozid“) zu bezeichnen. Siehe auch Catherine Coquio, „Violence sacrificielle et violence génocidaire“, in: Quasimodo, Nr. 8, 2005, S. 193-230; C. Coquio und A. Kalisky: „Vorwort“ zu L'Enfant et le génocide. Témoignage sur l'enfance pendant la Shoah, Paris 2007, S. VII-XCVI.

11 S. Felman: „Im Zeitalter der Zeugenschaft: Claude Lanzmanns Shoah“ (Anm. 4), S. 176. 
wieder unterminiert wird. Mit dem Versuch, jegliche Möglichkeit des Bezeugens der Realität des Verbrechens durch Geheimhalten des Tatorts, Vertuschen der kriminellen Handlung im öffentlichen Diskurs und physische Vernichtung der Zeugen $\mathrm{zu}$ verhindern, wird durch das staatlich organisierte Massenverbrechen eine Vereitelung des Bezeugens und schließlich eine Verleugnung des Zeugnisses an sich bewirkt. Das Paradox und die Spezifizität der modernen politischen Gewalt bestehen darin, dass die Zeugenschaft sich genau auf dieses Gesetz der Evidenz stützt, das sie angreift: Indem das Verbrechen oft nur noch durch die überlebenden Augenzeugen attestiert werden kann, ist seine Bezeugung ein umso prekärerer Prozess. Den Zeugnissen wird im Namen des Gesetzes der Evidenz aufgrund ihrer fehlenden Objektivität und Zuverlässigkeit der mangelhafte Status eines imperfekten Beweismittels verliehen.

Die Formen des kollektiven Gedächtnisses, die im Zusammenhang mit dieser Art von Massenverbrechen auftreten, werden demzufolge stark von der Konfrontation zwischen Zeugnissen von Opfern und dem Versuch, diese anhand von durchdachten Verleugnungsstrategien unglaubwürdig zu machen bzw. zum Schweigen zu bringen, bestimmt. Die Auswirkungen einer solchen Gewaltform auf das kollektive Gedächtnis müssen daher ausgehend von den Beziehungen zwischen dem Zeugnis als Wieder- und Weitergabe (oder Ver- und Übermittlung) der Erfahrung des Opfers der politischen Gewalt und seiner Negation ${ }^{12}$ untersucht werden. Diese Beziehungen decken sich teilweise mit jenen, die zwischen Wahrheit und Politik seit dem für die Moderne kennzeichnenden Aufkommen des nationalen und bürokratischen Staats existieren. Die politische Gewalt, wie sie von einem verbrecherischen Staat ausgeübt wird, impliziert immer einen Krieg gegen die Erinnerung und gegen das Zeugnis, einen Krieg, der aus der Etablierung der Lüge als Prinzip der Politik hervorgeht und somit eine Gegenrealität generiert. Diese Gegenrealität, wie sie beispielsweise vom ideologischen Negationismus verteidigt wird, kann zuweilen in demokratischen politischen Systemen deswegen weiterbestehen, weil wir es mit einer allgemeinen Auflösung der Beziehungen zur Wahrheit zu tun haben, die wiederum auf einer Konkurrenz zwischen unterschiedlichen Wahrheits- und Wissensordnungen gründet und deren Genealogie es zu verstehen gilt. Die Auflösung der Beziehungen zur Wahrheit sind dort besonders deutlich erkennbar, wo die von Arendt als „Tatsachenwahrheiten“13 bezeichneten Tatbestände zum Gegenstand

12 Auf die notwendige logische wie epistemologische Unterscheidung zwischen ,Verneinung (oder ,Negation'), ,Verleugnung', ,Verwerfung' und, Verdrängung' kann an dieser Stelle nicht ausführlich eingegangen werden. In Anlehnung an Freuds Unterscheidung zwischen, Verdrängung' und ,Verneinung' werden jedoch ,Negation', ,Leugnung' und ,Verleugnung' als aktive Positionen, welche die Übertretung einer positiven Norm einschließen, die mit der öffentlichen Bekanntmachung eines (juridischen, historiografischen, testimonialen) Urteils über die Vergangenheit einhergeht, aufgefasst werden. Siehe hierzu C. Coquio, „A propos d'un nihilisme contemporain“, in: Dies. (Hg.): L'Histoire trouée. Négation et témoignage, Nantes 2004, S. 29-89.

13 Siehe Hannah Arendt: Wahrheit und Lüge in der Politik, München 1997, S. 330; siehe auch Jacques Derrida: Prolegomena zu einer Geschichte der Lüge, Frankfurt am Main 1997. 
unterschiedlicher „Ansichten“ und lediglich zu „Meinungen“ erklärt werden. Beispielhaft dafür sind die immer wieder aufkommenden Debatten im öffentlichen Raum über den Genozid an den Armeniern und an den Herero und Nama, wie sie in den letzten Jahren in Deutschland geführt worden sind. ${ }^{14}$ Die Auflösung der Beziehungen zur Wahrheit liegt größtenteils an der mangelnden Autorität, die dem Zeugnis zugestanden wird - und das, obwohl wir uns schon seit einem Jahrzehnt in einem Kontext befinden, in dem das „Recht auf Wahrheit" durch die UNO als grundlegendes Menschenrecht definiert worden ist. ${ }^{15}$ Die Auswirkungen der vom Staat erzeugten historischen Katastrophen auf die kollektiven Erinnerungsformen, die alles andere als ein Epiphänomen sind, müssen in ihren Konsequenzen auf die Gesamtheit der Möglichkeiten, historisches Wissen und eine öffentliche Wahrheit zu produzieren, begriffen werden. Eine erneute Reflexion über den Stellenwert des Zeugnisses, das seit den 1990er Jahren sowohl in der Wissenschaft wie auch im Recht eine immer größere Bedeutung einnimmt, gehört unbestreitbar dazu. ${ }^{16}$

Zweifelsohne ist aber auch eine Reflexion über Formen von Zeugenschaft in der Kunst unerlässlich, da die Form des Zeugnisses und der Akt des Bezeugens sowie die Reflexion über Zeugenschaft auch in der Kunst ihren Ort gefunden haben. Gerade die Fülle an Zeugnissen, die schon unmittelbar nach dem Krieg publiziert worden sind, haben dazu geführt, dass die unerhörte Krise, die den Akt der Übermittlung von menschlicher Erfahrung und ihre mögliche Integration in objektivierende Wissensformen und -praxen im Kontext von extremer politischer Gewalt betrifft, überhaupt wahrgenommen werden konnte. ${ }^{17}$ So sind Hannah Arendts tiefgreifende Überlegungen zu der nationalsozialistischen KZ- und Gulag-Erfahrung und ihrer anthropologischen Folgen u.a. auf deren nahezu unmögliche Übermittelbarkeit durch die Augenzeugen paradoxerweise nicht ohne ihre Lektüre von literarischen oder nicht literarischen Zeugnissen und Berichten zu denken. ${ }^{18}$ Genauso wenig ist die Ernennung der Epoche nach 1980 zur „Ära des Zeugen“ durch Annette Wieviorka und Shoshana Felmans Schlagwort von der „Krise der

14 Siehe u. a. Yvonne Robel: Verhandlungssache Genozid: zur Dynamik geschichtspolitischer Deutungskämpfe, München 2013. Die Bezeichnung des Verbrechens an den Armeniern als „Völkermord“ durch Joachim Gauck zum 100. Gedenktag am 24. April 2015 und die jüngsten Äußerungen des Bundestagspräsidenten Norbert Lammert sowie der Bundesregierung zum „Völkermord“ an den Herero und Nama zum Anlass des 100-jährigen Gedenkens an das Ende der deutschen Kolonialherrschaft in Südwestafrika am 9. Juli 2015 deuten auf eine Wende im geschichtspolitischen Diskurs in der Bundesrepublik Deutschland hin.

15 Siehe: http://www.un.org/en/events/righttotruthday/documents.shtml.

16 A. Kalisky : „D’un savoir en crise à un savoir indiscipliné. Le statut du savoir de la victime de violence de masse dans les pratiques judiciaires et historiographiques“, in: Études arméniennes contemporaines, Nr. 5, S. 25-77.

17 Siehe hierzu Shoshana Felman und Dori Laub: Testimony: Crises of Witnessing in Literature, Psychoanalysis, and History, New York/London 1992.

$18 \mathrm{Zu}$ Arendts problematischer Beurteilung der Autorität der Zeugen aus den Konzentrationslagern und ihre frühe Beschreibung einer Krise der Zeugenschaft vgl.: A. Kalisky: „D’un savoir en crise à un savoir indiscipliné“, in: Études Arméniennes Contemporaines, Nr. 5, 2015, S. $25-$ 77 . 
Zeugenschaft" von der langen Rezeptionsgeschichte dieser Korpora zu trennen. Erst das langsame, allmähliche Eindringen dieser Zeugnisse in das kulturelle Gedächtnis ermöglichte, dass eine Krise der Zeugenschaft diagnostiziert werden konnte, nicht zuletzt, weil viele unter ihnen selbstreflexive Züge aufweisen, die Überlegungen über die Gegenüberstellung von Zeugnis und Verleugnung, und über die Notwendigkeit einer künstlerischen Form des Bezeugens beinhalten. Kunst war so schon - nicht nur im metaphorischen Sinne - inmitten der gewalttätigen Ereignisse eine der prominentesten Formen der Zeugenschaft. Unzählige, während des Genozids verfasste Gedichte, Romane, Theaterstücke und Tagebücher, die zugleich immer auch Tatbestände bezeugen, sowie Archiv- und Dokumentationsprojekte, die auch kulturelles, insbesondere literarisches Archiv sind, veranschaulichen diese Zusammenhänge.

Der Text von Abraham Levite stellt ein einzigartiges Untersuchungsobjekt für diejenigen dar, die begreifen wollen, was auf dem Spiel steht, wenn bestimmte menschliche Grundinstitutionen direkt bedroht sind: das Bezeugen als eine Form des Tatsachenbeweises sowie als eine Form der Vermittlung von Erfahrung, das kollektive Gedenken an die Toten und die Trauer als individuelle und kollektive Beziehung zum Tod. Abraham Levite liefert mit seinem Text eines der frühesten Beispiele für einen selbstreflexiven Umgang mit dem Akt der Zeugenschaft und dessen Transformation in und durch Kunst: Er versucht eine Art ars poetica zu definieren, die durch die Massenvernichtung in Auschwitz bestimmt wird. Diese ars poetica legt nicht nur fest, was Kunst und was Zeugenschaft zu leisten hat, sondern versucht auch, die Beziehung der beiden Pole als ein dialektisches Wechselverhältnis von einer Kunst des Erzeugens und einer Kunst der Zeugenschaft zu denken.

\section{Ein sonderbares Manifest}

Auf der Schwelle zum Tod nimmt sich Levite die Zeit, die Angst vor dem Vergessen seiner Existenz und vor der Leugnung seiner Erlebnisse zu beschreiben. Da er auch die Ungläubigkeit seiner Leser befürchtet und sich seines und seiner Gefährten Todes sicher ist, bekräftigt er: „Wir müssen selbst erzählen was uns betrifft“. Levite sehnt sich vor allem danach, das „Geheimnis“ des Lebens der Auschwitz-Häftlinge zu übermitteln. Dieses „Geheimnis“ des Lebens in Auschwitz gilt es zu verdichten, seine „blutige[n] Stücke“ einzusammeln, es dem „Abgrund“ zu entreißen und ein „Bild“ zu schaffen, um es „der Welt [zu] zeigen“.

Das Geheimnis, auf das sich Levite bezieht, liegt in den Anführungszeichen, die das Partizip ,gelebt' im Originaltext umschließen. So scheint die Bedeutung des Wortes durch die Anführungszeichen bedingt und aufgehoben zu sein - genau wie sein eigenes Leben, das, umgeben von Leichen, droht, der Vernichtung zum Opfer zu fallen. Der bevorstehende Tod des Autors hat im Gegensatz zum Leben in Auschwitz nichts Geheimnisvolles und erfordert keine Anführungszeichen. Die Dokumentation der Todesfabrik erscheint ihm aus diesem Grunde nicht notwendig, sein vorrangiges Interesse gilt nicht dem Sammeln von „Fakten und Ziffern“, 
"trockene[r] Dokumente“, die auch ohne die Opfer einst von Juristen und Historikern zusammengetragen werden. Vielmehr geht es ihm darum, zu schildern, was die „vom Leben vergessen[en] “ Gefangenen „,erinnerten und fühlten, dachten und redeten“ und wie ihr „Leben“ sich in ein „Durcheinander von Leben und Tod“ gewandelt hat. Der Sammelband wird als kollektive Schreibform konzipiert, wie es das „wir“, das im Text so auffallend häufig gebraucht wird, aufzeigt. Bevor sie aus dem Leben scheiden, streben die Autoren des Bandes danach, darzustellen, was unter den anormalen Überlebensumständen eines Vernichtungslagers Normalität bedeutet.

Dafür ist es notwendig, diesem durch den Tod deformierten Leben Form zu verleihen und der Welt seinen menschlichen Charakter zu offenbaren, wofür Levite eine poetologische Leitlinie verfasst. Diese Leitlinie ist jedoch von Widersprüchen geprägt: Die Form, in der das Geheimnis übermittelt wird, ist genau genommen weder Zeugnis noch Dokument, gehört jedoch ebenso wenig unmittelbar zur Literatur. So präzisiert Abraham Levite selbst, dass, auch wenn der Sammelband nicht darauf abzielt, „die Geschichte von Auschwitz zusammen[zustellen]“ wie „Zeugnisse und Dokumente" es tun würden, die Texte seiner Autoren genauso wenig ,auf der literarischen Waage gewogen werden" könnten. Die implizite Definition, die Levite vom ,Zeugnis' liefert, muss als Schilderung oder Bericht einer Tatsache (der Massenvernichtung) oder Beschreibung einer Situation und eines Ortes (des Konzentrationslagers) verstanden werden, die von einem direkt betroffenen Zeugen durchgeführt wird und an einen Leser bzw. Zuhörer außerhalb dieses Ortes und seiner Geschehnisse gerichtet ist. Dieses Verständnis von Zeugnis als Schilderung oder Beschreibung zieht seine Autorität aus der Anwesenheit des Autors als Augenzeuge beim geschilderten Ereignis und am beschriebenen Ort. Die Definition entspricht dem Begriff des epistemischen ,Zeugnisses', wie er in der westlichen Erkenntnistheorie seit dem 18. Jahrhundert etabliert worden ist.

Auch wenn Levite die Texte der Anthologie nicht als Zeugnisse im epistemischen Sinne definiert, stuft er sie ebenso wenig als Dokumente ein. Der Begriff ,Dokument', der viermal im Text auftaucht, zeigt sich im Grunde genommen als konträr zu der Form, die Levite zu definieren sucht. Zunächst entziehen sich die Texte der Opfer der Vorstellung von „vollständigen Dokumente[n]“. Im Gegensatz zum unvollständigen Charakter des Fragments oder der „Bröckchen“ der Texte der Opfer haben Dokumente die Eigenschaft, einen Aspekt der Realität gründlich darzustellen und Informationen über diese Realität zu liefern, die durch einen wissenschaftlichen, objektivierenden Zugang ausgewertet werden müssen. Gleichzeitig kann das „vollständige“ Dokument aber auch auf das angestrebte Ziel anspielen, auf die Erfüllung eines Versprechens oder einer Pflicht der Übermittlung, die im Fall des Sammelbandes Auschwitz keineswegs garantiert ist, da ihre Autoren zu Tode verurteilt sind. Der Begriff des „trockenen“ Dokuments, gegen den sich die Texte von Levite und seiner Gefährten stellen, suggeriert einen höheren Grad an Objektivität: Ihm wird auf epistemischer Ebene eine größere Autorität zugesprochen als dem Bericht des Zeugens, dessen Autorität sich einzig aus seiner Gegenwart an Ort und Stelle speist. Im Gegensatz zur Subjektivität des Zeugnisses ist der 
Begriff des Dokuments leicht mit Schriftstücken in Verbindung zu bringen, die von den SS-Verwaltern des Lagers stammen und nicht vollständig zerstört werden konnten (wie Listen von Toten und Gefangenen, Pläne der Krematorien und Baracken). Aber gerade weil ihm die Subjektivität der Opfer fehlt, scheint das Dokument gewissermaßen auf der Seite des Todes zu stehen: Es gibt vor allem wieder, „wie in Auschwitz gestorben wurde“. Demgegenüber vermag einzig der Text des Opfers in seiner Unvollständigkeit, das Leben in Auschwitz als „Durcheinander von Leben und Tod" zu beschreiben. Und doch bekräftigt Abraham Levite zum Ende seiner Einleitung, dass die Texte der Opfer ,als Dokument“ zu lesen sind und wirft damit die Frage auf, ob dem unvollständigen und subjektiven Bild so viel Wert wie dem Dokument beigemessen werden sollte.

Das Leiden der Gefangenen übersteigt jegliches Vorstellungsvermögen. Abraham Levite selbst hebt hervor, es würden Mittel fehlen, dieses Leid adäquat auszudrücken. Das „Geheimniss“ des Lebens dieser Menschen beruht auf einer Erfahrung, die selbst völlig irreal erscheint. Und doch ist diese Erfahrung real, sie ist nicht eine imaginierte Fiktion. Aus diesem Grunde mutet die Darstellung durch mimetisches Spiel als problematisch an, da sie gleichzeitig künstlerisches Spielen zu verbieten scheint. Wie es Levite selbst formuliert, besteht eine Diskrepanz zwischen der wirklichen Erfahrung und der Erfahrung, wie sie durch die Kunst vermittelt wird: Der Künstler leidet nicht wirklich, „[d]enn ihm tut ja nichts weh“. Er kann das Leiden nur nachahmen. Im Gegensatz dazu existieren in Auschwitz keinerlei „Posen und Getue“. Also muss man sich eine Form von Kunst vorstellen, die nicht spielt, die nicht nur ästhetisches Vergnügen sein will, sondern eine Kunst, die eine Wahrheit vermittelt und die Sprache der Wahrheit spricht.

Paradoxerweise ist die Form, die hier gesucht wird, ein Mittel, um eine höhere Wahrheit zu vermitteln, die über der Kunst selbst steht und die den Regeln der Kunst, die von der bisher bekannten Literatur aufgestellt wurden, nicht gehorcht. Im Unterschied zur Tragödie, die durch auf der Bühne simuliertes Leiden beabsichtigt, Katharsis hervorzurufen, ist das Schreiben der Zeugen von Auschwitz aus der Sicht Abraham Levites nicht der Ausdruck fiktiver Handlungen, sondern erlebter Erfahrung und realen Leidens. Genau deswegen verursacht ihre Darstellung jenes „authentische Unbehagen“, von dem David Hume spricht. ${ }^{19}$ Gleichzeitig lässt die angestrebte Schreibform jedes „bereitwillige Ausklammern des Zweifels" obsolet werden, das heißt jegliches "Vertrauen in die Dichtung" in dem Sinne, wie es Samuel Taylor Coleridge für fiktionale Werke definiert hat. ${ }^{20}$ Der testimoniale Aspekt der Schreibform hebt jede ästhetische Urteilskraft im Sinne von Kants Interessenlosigkeit gegenüber der realen Welt auf. Ein Autor, der auch Opfer oder Überlebender ist, verlangt also mehr vom Leser als ein einfaches Fürwahrhalten, er

19 „It is certain, that the same object of distress which pleases in a tragedy, were it really set before us, would give the more unfeigned uneasiness [...]“, David Hume: Four Dissertations, III. Of Tragedy, London 1757 [reprint New York 1970], S. 188, von mir hervorgehoben.

20 Samuel Taylor Coleridge: Biographia Literaria, J. Shawcross (Hg.), Oxford 1965, Bd. II, S. 6. 
verlangt, dass man ihm glaubt, ohne dass dieser Glaube bloß ein ästhetischer zu sein hat.

Levite weiß, dass die Opfer ihre Erfahrung nicht allein durch das Kunstwerk übermitteln können. Seit Aristoteles wird die literarische Kunst oder poiesis als ein verbaler schöpferischer Akt verstanden, der sich in unterschiedliche Modi unterteilt, die die Wirklichkeit nachahmen und somit dem von Herodot definierten legein ta onta (das Sagen, was ist) entgegengesetzt ist. Im Falle der in Auschwitz verfassten Schilderungen von Erfahrungen muss die Vermittlung mithilfe der Kunst zwar über die Imitation der Realität erfolgen, dabei jedoch mehr als bloßes Spiegelbild der Realität sein. Die Realität muss (wieder)erschaffen werden. Die im Text beschriebene hybride Form zeichnet sich also durch eine fast paradoxe Gegensätzlichkeit aus, indem sie sich deutlich sowohl vom Zeugnis - als direkte Wiedergabe einer gelebten Erfahrung - als auch von einem literarischen Werk unterscheidet und die beiden Dimensionen von legein und poien in sich vereint. So schreibt Levite, es sei notwendig, „alles aufzuschreiben“ und zugleich „etwas zu schaffen“, das im Stande ist, die Tragödie der conditio humana im Lager „aus[zu]drücken und $\mathrm{ab}[\mathrm{zu}]$ spiegeln".

Wir sehen uns also mit einer paradoxen Definition konfrontiert, einer Definition, die das Zeugnis zwischen unmittelbarer Wiedergabe von Erfahrung und Literarisierung situiert. Die von Levite definierte Schreibform weist Merkmale beider Aspekte auf, weil sie als Dokument verstanden und zugleich eindeutig „literarisch genommen" werden muss - wenn auch, um sich gerade von der literarischen Tradition abzuheben. Dabei soll das Schreiben keinesfalls auf Kunstgriffe und fiktionalisierende Modi verzichten, sondern die Beziehungen zwischen Authentizität der Erfahrung und Literarisierung neu definieren. Im Dienste einer lebenswichtigen Aufgabe verändert sich die poetische Kunst durch die singuläre Erfahrung im Vernichtungslager tiefgreifend und verfügt dabei nur über einen beschränkten Spielraum. Das Werk zeigt eine neuartige poetische Sprache, die schöpferisch ist, ohne sich jedoch in den Dienst der Fiktion oder des Gedichts zu stellen: Ganz im Gegenteil, es sind die Fiktion oder das Gedicht, die der Wahrheit, die es zu übermitteln gilt, zu Diensten stehen. Die Umschreibung der vom Zeugen angestrebten Form nimmt somit die Züge einer dialektischen Gratwanderung an, die sich zwischen Zeugnis (bzw. Dokument) und Kunst bewegt.

In diesem Sinne kann Levites Text als literarisches Manifest gelesen werden. Eine neue Literatur wird definiert, die nicht als „Dokument“, sondern als „Fragment" bezeichnet wird, das wiederum rezeptionsästhetisch "wie ein Dokument" betrachtet werden muss. Denn diese Art von Literatur „wie ein Dokument“ aufzufassen, bedeutet, zur Beurteilung des „künstlerischen Wert[s] der Sache“, „Zeit und Ort“ zu berücksichtigen und die Einzigartigkeit der Erfahrung mit auf die „literarische Waage" zu legen. Die Beurteilung eines literarischen Werts ist hier von der Erfahrung des Autors nicht zu trennen, denn wie könnten wir „das Opfer dafür kritisieren, dass es zu laut stöhnt oder zu leise weint"? Es handelt sich somit schließlich um ein „Zeugnis“ („wir wollen Zeugnis ablegen“), dessen testamentarischer Sinn und Wert im Entstehungsort und in der Zeit seines Schreibens liegen, die 
untrennbar mit der Erfahrung verbunden sind: „Und die Zeit ist: kurz vor dem Tod; und der Ort ist: auf dem Schafott". In vier Takten, in einem unerbittlichen Rhythmus, ist alles gesagt.

Literatur als Kunst der Übermittlung von einer singulären menschlichen Erfahrung des Unmenschlichen erfordert somit eine neue literarische Ausdrucksweise, welche die Literatur- und Kunstgeschichte gleichzeitig aussetzt und neu definiert. Damit wird ein Gründungsgestus manifest, der sich bei Autoren, welche die NSLager oder den Gulag überlebten, wiederfinden lässt und den Catherine Coquio als einen „literarischen Schisma“ benennt. ${ }^{21}$ Bei einem Zeugen in Auschwitz stellen allerdings diese paradoxe Einstellung gegenüber der Literatur und dem Bezeugen sowie diese Forderung nach einer klaren Zäsur in der Kunstgeschichte etwas Unerwartetes dar. Ein Zeuge aus Auschwitz fordert nichts weniger als ästhetische Distanz einzunehmen gegenüber der erlebten Gewalt, und dies unmittelbar am Ort und in der Zeit des Geschehens! Hingegen klammern die meisten Theorien über frühe, inmitten des historischen Geschehnisses entstandene Zeugnisse, den literarischen, und erst recht den fiktionalisierenden Gestus derselben aus. Dem Zeugen inmitten des Ereignisses geht es, gemäß dieser Theorien, vielmehr um das Bezeugen von Fakten und um die Mitteilung von Erlebtem, die der Etablierung eines objektiven Wissens über historische Tatsachen oder über das Verbrechen im juridischen Sinn nutzen kann. Infolgedessen wurde in der Literaturtheorie ein Narrativ etabliert, das Literarisierung - und in erster Linie Fiktionalisierung - von zeitlicher und räumlicher Distanz abhängig macht. ${ }^{22}$ Dieser Auffassung nach sollen die ersten Zeugnisse, die am Ort und zum Zeitpunkt der Ereignisse und unmittelbar nach der Befreiung entstanden sind, einem strikteren autobiografischen Pakt gehorchen als die darauf folgenden Texte. Obwohl viele dieser Werke literarisch sind, werden sie demzufolge nur selten als Literatur per se untersucht. Und obwohl viele darunter eindeutig fiktional sind, werden sie dementsprechend aus der Analyse des Korpus der ,Zeugnisse' als hypothetische ,Gattung' ausgeklammert. ${ }^{23}$

Levite definiert allerdings die Texte der Anthologie Auschwitz als eine Form von Literatur, die Unmittelbarkeit fordert. Die Texte der Opfer sollten so bescheidene „Blättchen“, kurz und scharf sein: „[K]urz wie die Tage, die [ihnen] noch zum Leben bleiben, und scharf wie die Messer, die auf [ihre] Herzen zielen“. Diese For-

21 Siehe dazu ihren Text „La ,vérité du témoin comme schisme littéraire“, in: La Licorne, Nr. 51, Rennes 1999, S. 55-79. Siehe auch ihr jüngst erschienenes Buch La Littérature en suspens. Écritures de la Shoah: le témoignage et les oeuvres, Paris 2015.

22 Siehe hierzu z.B. der Sammelband von P. Kuon, S. Segler-Messner, M. Neuhofer (Hg.): Vom Zeugnis zur Fiktion. Repräsentation von Lagerwirklichkeit und Shoah in der französischen Literatur nach 1945, Wien 2006.

23 Siehe hierzu C. Coquio: „Le récit de rescapé est un genre littéraire` ou le témoignage comme ,genre de travers'?“, in: La Licorne, Nr. 82, 2007, S. 103-132; A. Kalisky: „Les voix distantes. Réflexions sur les malentendus du témoignage“, in: Ruth Vogel-Klein (Hg.): Die ersten Stimmen. Les Premières voix. L'écriture de la Shoah en langue allemande, 1945-1963, Würzburg 2010, S. 289-328. 
derung nach Nüchternheit wird am Ende des Textes mittels einer Allegorie beschrieben:

„Schweigen, das tun nur die Bonzes. Die machen ein geheimnisvolles Gesicht, als hätten sie wer weiß was zu sagen. Aber erst dort in der Welt der Wahrheit, wo Pose und Getue nichts mehr gelten, kommen sie mit dem Geheimnis ihres Lebens heraus: einem Brötchen mit Butter."

Die Figur des Bonze taucht häufig in der jüdischen Folklore und in der jiddischen Literatur Osteuropas auf, wie insbesondere in der Erzählung „Bonze der Schweigsame“ oder „Bonze Schweig“ (1894) von Jizchok Leib Perez. ${ }^{24}$ Diese von Perez wohl bekannteste Erzählung schildert wie Bonze im größten Elend lebt, doch, im Gegensatz zu Hiob, nie darüber klagt und immer wieder schweigsam sein Leid hinnimmt: „Er schwieg auch im Spital, wo man doch schreien darf! [...] Er schwieg in der Agonie, er schwieg im Sterben... Kein Wort gegen Gott, kein Wort gegen Menschen! ${ }^{‘ 25}$. Erst im Jenseits ist er bereit, das „Geheimnis [sein]es Lebens" durch einen Wunsch zu enthüllen: „eine warme Semmel mit frischer Butter“. Die Bescheidenheit und Inkongruenz seines Wunsches beschämen die Engel und den Richter und bringen den Ankläger zum Lachen. „Still geboren, still gelebt, still gestorben und noch stiller begraben “26, steht Bonze in Perez'Erzählung für das Massenschicksal der Armen. Nach seinem Tode erhält er nicht einmal ein Grab, da der Wind die Holztafel mit seinem Namen umwirft und bereits drei Tage nach seinem Ableben der Totengräber vergessen hat, wo er ihn begraben hat. „Wie ein Schatten glitt er durch unsre Welt [...]", schreibt Perez:

„Ein Schatten! In keinem Menschenherzen, in keinem Menschenhirn blieb Bonze Schweigs Bild zurück. Nichts erinnert an ihn. Elend gelebt, elend gestorben!“ ${ }^{\prime 27}$

Der Gefangene in Auschwitz aber möchte nicht schweigend sterben, nicht in die sofortige Vergessenheit geraten. Obwohl er weiß, dass er wie Bonze kein Grab haben wird, will er im Gegensatz zu ihm einen Text verfassen, der, gleich einem Schrei, die „eiskalte Gleichgültigkeit der Völker“ erschüttern kann. Die Welt der Wahrheit darf nicht nur das Jenseits sein, die Wahrheit muss sich auch im Diesseits offenbaren. So spricht der Allbarmherzige selbst zu Bonze vor dem göttlichen Gericht: „Vielleicht wusstest du sogar selbst nicht, dass du schreien kannst, und dass vor deinem Schreien die Mauern Jerichos erzittern und einstürzen würden $[\ldots]^{“ 28}$. Mit seinem literarischen Vorhaben will der zum Autor gewordene Häftling das Schweigen brechen, auch wenn er sich am Rande des Todes befindet. Obwohl er sich auf der literarischen Ebene für „stotternd“ hält, unterstreicht er die

24 Jizchok Leib Perez: „Bonze Schweig“, in: Jüdische Geschichten, aus dem Jiddischen übertragen von Alexander Eliasberg, Leipzig 1923, S. 50-59.

25 Ebd., S. 57.

26 Ebd., S. 52.

27 Ebd., S. 51.

28 Ebd., S. 58. 
Notwendigkeit, die Erfahrung in Auschwitz ,in unserer Sprache“, das heißt in der Sprache der Opfer, zu schildern.

Die erwünschte literarische Form beinhaltet eine testamentarische Dimension: Sie soll den letzten Wunsch der „lebendige[n] Tote[n]“ von Auschwitz erfüllen, nämlich die Tradierung an die ,am Leben gebliebenen freien Brüder“, die vielleicht etwas davon lernen können. Dieser Wunsch kann nur durch die Erschaffung einer Form nachgekommen werden, welche die Literatur im Angesicht der Erfahrung der Katastrophe neu definiert. Wie bereits aufgeführt, darf die Repräsentation des Leidens der Opfer sich nicht in bloße ästhetische Ergötzung wandeln. Diese Forderung lässt sich auch als eine Reflexion über das Wesen der durch die Kunst ausgedrückten Trauer verstehen:

„[...] jeder kultivierte und anständige Mensch [wird es] für seine Schuldigkeit ansehen, uns zu bedauern und Nachrufe auf uns zu halten. Wenn unsere Schatten auf Leinwänden und Kanzeln erscheinen, werden mitleidige Damen sich mit ihren parfümierten Taschentüchern die Augen wischen und uns beklagen: ach, ihr Unglücklichen!“

Auch wenn Levite von einer „tragische[n] Generation“ und „tragischen Bedingungen" sowie von einer ,Tragödie" spricht, drückt sich damit seine Ablehnung der Katharsis aus. Er verweist darauf, dass das Massenschicksal der Gefangenen von Auschwitz nicht in die Tradition der literarischen Tragödie einzuschreiben sei. Die Erfahrung der Trauer mittels Kunst ist von nun an verunmöglicht. Genauso begrenzt ist jede empathische Haltung den Opfern gegenüber, weil die Diskrepanz zwischen ihrer Erfahrung und jener des Rests der "freien“ Welt unüberwindbar ist, sogar (oder gerade) mittels der Imagination. Auf den ersten Blick scheint Levite die Begriffe ,Zeugnis' und ,Literatur' abzulehnen, doch geht es ihm vielmehr darum, sie seinen eigenen Bedingungen zu unterwerfen und sie konzeptionell neu zu definieren.

So scheint es, dass der Text eine in sich widersprüchliche, zugleich verbindende und dialektische Form definiert, die sowohl der, Klage - welche die Trauer die dem in Literatur gewandelten Zeugnis inhärent ist, ausdrückt - als auch der ,Anklage die sich auf Dokumente stützen kann - eine Stimme verleihen soll. ${ }^{29}$ Die Texte können als anklagende Klage gelesen werden, die sowohl an die freie Welt in ihrer Gleichgültigkeit als auch an Gott in seiner (Un)Gerechtigkeit appelliert. Eindeutig Teil der jüdischen Kultur, aber ebenso der christlichen und, in einem weiteren Sinne, der westlichen - darauf deutet die intertextuelle Referenz auf den Dritten

29 In seinem Karl Kraus gewidmeten Text führt Walter Benjamin die Unterscheidung zwischen der ,Klage‘ und ,Anklage‘ ein. Sigrid Weigel nimmt diese Differenzierung für ihre Reflexionen über den Status des Zeugnisses in Recht, Geschichte und Literatur wieder auf: „Zeugnis und Zeugenschaft, Klage und Anklage. Die Geste des Bezeugens in der Differenz von, identity politics", juristischem und historiographischem Diskurs", in: Einstein Forum Jahrbuch, Berlin 1999, S. 111-135. 
Gesang in Dantes Divina Commedia hin ${ }^{30}$-, demonstriert der Text gleichzeitig den Verfall seines gesamten kulturellen Erbes, indem er die Möglichkeit der Trauer durch die Kunst ablehnt. Er kann also nicht allein als Manifest einer neuen literarischen Kunst verstanden werden, die der Zerstörung des Zeugnisses entgegenwirkt, sondern auch als Manifest einer neuen Kunst der Trauer, die sich der Unterbindung der Trauer, die jedem Völkermord innewohnt, widersetzt.

\section{Das Zeugnis als Erzeugnis}

Genauso wie die im ewigen Eis gefangenen Polarreisenden ihre Stifte nicht aus der Hand legen, schreibt der in Auschwitz Gefangene Levite bis zu seinem Tode in Blick auf die „Ewigkeit“. Sich „über [sein] Schicksal erh[ebend] “ möchte er „etwas hinterlassen", eine Spur, auch wenn sie nur ein Fragment, ein Stottern ist. Sein Schreiben versetzt ihn in eine Zeitlichkeit, die das Menschliche überschreitet und das Göttliche berührt. Die Texte der Anthologie Auschwitz sind so auch Ausdruck einer messianischen Hoffnung, die trotz allem erhalten bleibt. Das Schreiben eines „Bekenntnisses“ richtet sich an die zukünftigen Menschen, das Zeugnis-Ablegen wird zum Schma Jisrael „vor den kommenden Generationen“ und für die „am Leben gebliebenen freien Brüder". Der zum Autor gewordene Zeuge weiß, dass er seine Aufgabe in einer dreifachen Zeit erfüllen muss, die sich aus der göttlichen Zeit der Schöpfung, die dem endlosen Fall des Menschen gegenübersteht, der natürlichen Zeit der Menschheit und der unmenschlichen Zeit des in Auschwitz zum Tode Verurteilten, konstituiert. ${ }^{31} \mathrm{Um}$ das Leiden auszudrücken, greift Levite auf Metaphern der talmudischen Klage zurück und knüpft für die Bezeugung der Geschehnisse paradoxerweise wieder an die Kunst in ihrer engen Verbindung zu Trauer und Leid an. Um die erlebte Erfahrung zu übermitteln, muss dem Text auch auf seiner faktischen Ebene geglaubt werden. Indem er das reine Dokument und das Zeugnis aber ablehnt und Anspruch auf seine eigene Schöpfungskraft erhebt, um der stotternden "Stimme des Weinens" in der freien, nachkommenden Welt

30 Im Dritten Gesang des Inferno gelangen Dante und sein Führer Virgilio an die Tore der Hölle unterhalb der Stadt Jerusalem, auf deren Giebel geschrieben steht: „Lasciate ogne speranza, voi chi'intrate“ („Lasst, die ihr eintretet, alle Hoffnung fahren“). Die intertextuelle Anspielung auf Dantes Inferno kommt in den Zeugnissen und Texten aus den Konzentrationslagern der Nazis häufig vor. Der in diesem Sinne wohl bekannteste Text ist das Kapitel „Der Gesang des Odysseus" aus Primo Levis Ist das ein Mensch?, das dieselben Verse aus Dantes Inferno zitiert.

31 Über die Wiederaufnahme der talmudischen Tradition der Klage in der Literatur aus der Shoah vgl. Christina Pareigis: „Die Klage erreicht uns nicht aus einer gewesenen Gedenkschrift. Zur biblischen und talmudischen Schreibweise in der jiddischen Lyrik Kadye Molodovskys und Mordechaj Gebirtigs im Angesicht der Shoah“, in: Karol Sauerland, Ulrich Wergin (Hg.): Literatur und Theologie. Schreibprozesse zwischen biblischer Überlieferung und geschichtlicher Erfahrung, Würzburg 2005, S. 197-213. 
Gehör zu verschaffen, wird der Text somit zum Archiv des Schmerzes oder zum „Schmerz-Archiv“.

Eingespannt in ein System von Gegensätzen kann sich die Literatur, wie sie hier im Dienste des Bezeugens einer Wahrheit der Erfahrung definiert wird, also nur durch einen multidimensionalen Akt verwirklichen. Der Text und die Anthologie, die er einleitet, müssen als objektive Dokumente betrachtet werden, auch wenn sie sich von einer Zeugenaussage vor Gericht deutlich unterscheiden. Nichtsdestoweniger sind sie auch in ihrer Materialität gewissermaßen Beweise, sogar forensischer Art, die der Anklage der Täter dienen können. Sie müssen aber auch als anklagende Aussagen gegen Gott verstanden werden und gleichzeitig als Klagen, die sich an Gott richten und die Verbrechen vor der Menschheit enthüllen. Diese Klagen erinnern in ihrer Form an ein Testament, was sich an Ausdrücken wie "Schma Jisrael [...] vor den kommenden Generationen " erkennen lässt. Diese Zusammenhänge werden aber vor allem durch das Anrufen des Schicksals deutlich, mit dem der Text schließt und das sich indirekt auf den Bund mit Gott (das testamentum der Vulgata) bezieht. Die Assoziation mit der Figur des Märtyrers nimmt in diesem Zusammenhang eine besondere Rolle ein. Schließlich verbindet sie sowohl die Situation der Juden in Auschwitz, deren „Zeugnis“ zum „Bekenntnis einer tragischen Generation" wird, mit dem Schicksal der christlichen Märtyrer, die für ihren Glauben sterben als auch mit dem der Juden, die für die ,Heiligung des Namens' (,KidduschHa-Schem') sterben. Diese Verknüpfung ist aber keinesfalls eine Gleichsetzung, denn weder der ,Kiddusch-Ha-Schem' noch der ,Kiddusch-Ha-Chaim' (,Heiligung des Lebens') können an einem Ort, an dem Leben und Tod radikal verzerrt werden, noch ihren religiösen Sinn beibehalten. Gleichzeitig führt der Begriff des ,Martyriums' auch die semantische Tiefe des Begriffs ,Zeugnis' mit sich, zu dessen etymologischen Wurzeln der altgriechische Terminus martys zählt. Auch der Vergleich der Situation der Auschwitz-Gefangenen mit den Polarreisenden verweist auf einen spezifischen Kontext. Die Erforscher des geografischen Endes der Welt sind Gefangene, die mit ihrer Augenzeugenschaft und in Form von Berichten, Logund Tagebüchern dem Rest der Menschheit mitteilen wollen, was sie sahen. Der Beginn des Textes bezieht sich so auf das Tagebuch des Forschers Robert Falcon Scott, der 1912 während einer Expedition in der Antarktis an Hunger und Kälte verstarb und dessen Tagebuch Monate später gefunden und in mehreren Ländern Europas veröffentlicht wurde. ${ }^{32}$ Sein Tagebuch ist gleichzeitig die Schilderung einer Forschungsreise an die Grenzen des Planeten und das Testament eines Mannes, der sich seines Todes sicher ist.

Indem der Text Levites ein dichtes Netzwerk aus Vergleichen, Metaphern und intertextuellen Referenzen bildet, scheint er die dem Akt des Bezeugens inhärenten Bedeutungen und Dimensionen zu vervielfältigen: Er ist Reisetagebuch eines Augenzeugen, eine potentielle Zeugenaussage vor Gericht, Dokument, Beweismittel, Archiv, religiöses Zeugnis eines Märtyrers, Testament, Bekenntnis (sowohl im religiö-

32 Robert Falcon Scott: Letzte Fahrt. Kapitän Scotts Tagebuch 1910-1912 - Tragödie am Südpol, Wiesbaden 2012. 
sen Sinne als auch in Bezug auf die autobiografische Gattung der ,Bekenntnisse', die confessiones), Lektion und Klage in einem. Dadurch, dass er all dies innerhalb einer reflektierten Form des Schreibens zusammenführt und dabei trotzdem explizit ausdrückt, worin er sich von einigen dieser Gattungen, Begriffe und intertextuell zitierten Werke unterscheidet, scheint der Text seine Wirkung zu verstärken, um genau die Vermittlung, die er sucht, zu erreichen. Entgegen dem Anschein einer vermeintlichen Unvereinbarkeit der zahlreichen aufgeführten oder implizit gedeuteten Schreib- und Übermittlungsformen muss hier eine Vielschichtigkeit berücksichtigt werden, durch die unterschiedliche Aspekte des Phänomens und des Begriffs der Zeugenschaft aufgeworfen und eine ganze Palette testimonialer Akte und Gesten offengelegt wird. Dies betrifft sowohl die diachronen Variationen von Zeugenschaft, die sich im Laufe der Geschichte herausgebildet haben, wie auch seine synchronen Spielarten und damit die unterschiedlichen Formen von Zeugenschaft, die in derselben Zeit über dieselben Geschehnisse entstehen können. Diese Vielschichtigkeit zeigt sich besonders deutlich an der oxymorischen Formulierung eines „Schmerz-Archivs“.

Abraham Levite schreibt: „Sollen ein paar Blättchen fürs YIVO bleiben, fürs jüdische Schmerz-Archiv, sollen es unsere am Leben gebliebenen freien Brüder lesen; und vielleicht werden sie davon auch etwas lernen." Dazu bestimmt, heimlich aus dem Lager gebracht oder nach dessen Zerstörung im Boden gefunden zu werden, sind die Manuskripttexte der Anthologie Auschwitz als Archiv - vorerst im wortwörtlichen Sinn, weil ja das YIVO-Archiv von Levite genannt wird - bestimmt. Die lebendigen Dokument-„Bröckchen“, in denen die Autoren das Leben der Juden inmitten der Auslöschung des Judentums beschreiben, werden ihren Platz zwischen Tausenden von anderen Archivdokumenten über die jüdische Kultur und das jüdische Leben erhalten. Aber mit dem Oxymoron „Schmerz-Archiv“ bezieht sich Levite auf eine doppelte Tradition. Der Ausdruck kann tatsächlich für die Tradition jüdischer Geschichtsschreibung der Verfolgung stehen, die in einem Geschichtskonzept, das eng mit der Erinnerung verbunden ist, die Aufgabe des Gedenkens an die Leiden erfüllen muss und so dem Gebot ,Zachor '(,Erinnere dich!') entspricht. Die Texte der Anthologie sind also für ein Archiv des kulturellen Gedächtnisses bestimmt. Um seinem Text einen spezifischen Moment in der Geschichte und der Erinnerung zuzuschreiben, bezieht sich Levite auf die Tradition der Geschichtsschreibung in all ihrer vormodernen Komplexität und verleiht ihm somit einen reflexiven Charakter, den David Suchoff als eine Form "kritischer Geschichte“ avant la lettre interpretiert. ${ }^{33}$ Indem Levite den Begriff ,Archiv' gebraucht, ist er sich bewusst, dass diese Tradition gleichzeitig eine neue Praxis in der Geschichtsschreibung darstellt, und zwar die des Sammelns und Speicherns im Archiv gemäß einem modernen Verständnis: In der modernen Geschichtsschreibung werden die Geschehnisse nicht als zyklische Wiederkehr der Vertreibung seit der Zerstörung des Tempels aufgefasst. Vielmehr nehmen sie ihren Platz in einer linearen, historischen und profanen Zeit ein, die

33 David Suchoff und Pavel Polian (Anm. 2) sind vermutlich die einzigen Wissenschaftler, die Abraham Levites Text bis dato kommentiert haben. Siehe „A Yiddish Text from Auschwitz: Critical History and the Anthological Imagination“, in: Prooftexts, Nr. 19, 1999, S. 59-69. 
prinzipiell nicht den „Schmerz“ und das „Weinen“ berücksichtigen muss und die Erinnerung des verfolgten Kollektivs als subjektive (kollektive) Erfahrung eher in den Hintergrund rücken lässt. ${ }^{34}$ Hier sei an Jizchok Leib Perez', Jacob Dinezons und Schlomo (An-Ski) Sanwel Rappoports Aufruf aus dem Jahre 1915 erinnert, mit dem sie mitten im Ersten Weltkrieg die Intellektuellen, aber ebenso die Gemeinschaft aller jüdischen Gemeinden und Menschen dazu aufforderten, so viel dokumentierendes Material wie möglich über die Teilnahme der Juden am Krieg und über ihre Erfahrung der antijüdischen Verfolgung zu sammeln:

„Woe to the people whose history is written by strange hands and whose own writers have nothing left but to compose songs of lament, prayers and dirges after the fact. ${ }^{\text {35 }}$

Bei Levites Text handelt es sich jedoch um die ,hybride“ Form eines „SchmerzArchiv[s]". Einerseits wird damit das Archiv als Dokumentation oder sogar als dokumentarischer Beweis des Geschehenen erachtet, andererseits als Erinnerung an das Leiden und womöglich auch als Trauer, die später durch den Text zumindest teilweise ermöglicht sein wird. In diesem oxymorischen Begriff sieht man also deutlich, wie sich Traditionen, Absichten und Formen der Vermittlung der historischen Erfahrung verdichten und gegenüberstehen, die vom Rezipienten ein Verständnis erfordern, das sich in die jüdische religiöse Tradition einordnet und zugleich von ihr abhebt. In diesem Sinne ist auch das Gebet zu lesen, mit dem der Text schließt. Es richtet sich nämlich genauso an Gott wie an das Schicksal: „Es sei der Wille bei Dir / Nicht hört Er die Stimme des Weinens". Gott scheint durch das Schicksal ersetzt und wird damit zugleich von den Menschen bestimmt. Es handelt sich dabei auch nicht mehr nur um das Archiv, das übermittelt werden soll, sondern um die Klage der Opfer, „die Stimme des Weinens“, die niemand hört. Dort, wo in der Tradition des ,Zachors', die Erinnerung und ihre Weitergabe durch die liturgische Wiederholung der heiligen Texte auf eine messianische Zeit abzielen, in der Gerechtigkeit eintreten würde, läuft hier in Auschwitz die Weitergabe der Erinnerung der im Vernichtungslager Gefangenen von Anfang an Gefahr, vergessen, ja viel schlimmer, verleugnet zu werden. Daher kann sich die Übermittlung nur durch ihren ,Tikkun' retten: Sie wird einer religiösen Tat gleichgesetzt, die in der kabbalistischen Tradition eine Wiederherstellung der Harmonie und eine Art Wiedergutmachung der bösen Taten anstrebt. ${ }^{36}$

34 Über das jüdische Konzept von Zeit vgl. Yosef Hayim Yerushalmi: Zachor: Erinnere Dich! Jüdische Geschichte und jüdisches Gedächtnis, Berlin: 1988, insbesondere Anm. 7, S. 113.

35 Jizchok Leib Perez, Jacob Dinezon und Schlomo (An-Ski) Sanwel Rappoport: „Appeal to collect material about the World War", in: Haynt, 1. Januar 1915, wieder aufgelegt in: David Roskies: The Literature of Destruction. Jewish Responses to Catastrophe, Philadelphia 1988, S. 209-210.

36 Die Erwähnung der „Gefäße des Seins“ in Levites Text stellt eine Anspielung auf die Kabbala dar und führt einen Vergleich des Zeugnisablegens und des kabbalistischen „Tikkun Olam“ durch, auf die hier jedoch nicht ausführlich eingegangen werden kann. 
Die nachträgliche Aufnahme von Levites in Auschwitz verfasstem Text in das Jizkor-Buch seiner kleinen Heimatstadt ${ }^{37}$ ist daher eine Art ,Tikkun ' in Form einer Rettung des Andenkens an die Toten - selbst wenn diese sich auf die Rezeption der jüdischen Gemeinde beschränkt. Dies bedeutet jedoch nicht, dass der Text nur als Ausdruck einer Gemeinschafts- oder Kollektiverinnerung zu betrachten ist, die jedweder politischen Instrumentalisierung ausgeliefert werden kann. ${ }^{38}$ Das Personalpronomen ,wir', das den Text auffällig häufig durchwandert, ist dementsprechend nicht nur als jüdisches Opfer-Kollektiv in Auschwitz zu interpretieren, sondern verweist auch auf ein Kollektiv der Opfer von moderner politischer Gewalt. Ohne die jüdische Erfahrung zu universalisieren, geht es viel eher darum, zu erkennen, inwiefern die Bedeutung dieser bestimmten Erfahrung für die gesamte Menschheit prägend sein wird.

Etwas aus der von den Opfern übermittelten Wahrheit zu „lernen“, bedeutet in dieser Hinsicht, die ethischen sowie die politischen Implikationen jener Kunstform in Betracht zu ziehen, die den Opfern eine Stimme verleiht. Anhand konkreter Beispiele dieser Kunstform, wie Abraham Levites Text, wird deutlich, wieso die starren Oppositionslinien zwischen den verschiedenen Begriffen und Formen des Zeugnisses, aber auch zwischen politischen, ethischen, epistemologischen und ästhetischen Aspekten nicht aufrechterhalten werden können. So wird der Leser schließlich dazu aufgefordert, die Oppositionen in eine dialektische Bewegung zu bringen. ${ }^{39}$ Diese besondere Form, die sich jenseits der Typologien von Zeugenschaft situiert und das Schreiben als Gratwanderung zwischen Kunst und Zeugenschaft begreift, kann als eine ,Kunst des testimonialen Er-zeugens' bezeichnet werden. Das Wort ,Erzeugnis' könnte die hybride Form charakterisieren, die zugleich Fakten dokumentiert und belegt und eine subjektive Wahrheit des Zeugen vermittelt, die auf einer anderen Ebene als die faktische Wahrheit ansetzt. Um sie zu

37 Die „Jizkor“ oder „Yizkor-Bücher“ (aus dem Hebräischen ,Yizker': etwas oder jemandem ,gedenken') sind eine seit dem 13. Jahrhundert etablierte Tradition der jüdischen Gemeinden des Ostjudentums. Es handelte sich ursprünglich um eine religiöse Tradition eines Gedenkbuches, in dem die Märtyrer der Gemeinde, die durch die Christen für ihren Glauben ermordet worden waren, in einem gebetähnlichen Text genannt und aufgezählt wurden. Im Zuge der Säkularisierung wurde die Tradition im 19. Jahrhundert vor allem im russischen Kaiserreich aufgenommen, um den in einem Pogrom getöteten Juden zu gedenken. Die Tradition wurde dann während und nach der Shoah weitergeführt, um den zum Teil vollkommen verschwundenen jüdischen Gemeinden einen Ort im kollektiven Gedächtnis zu verleihen. Hier handelt es sich dabei oftmals um Sammelbücher, die ganz unterschiedliche Textarten beinhalten (Beschreibungen des Lebens der Gemeinde vor dem Krieg, Schilderungen der Ereignisse während des Genozids etc.). Vgl. „Jizkor-Buch“, in: Enzyklopädie des Holocaust, München/Zürich 1995, Bd. II, S. 677-679.

38 So wie es im Umgang mit Levites Text im Rahmen der israelischen Erinnerungspolitik deutlich wurde. Siehe meinen Text „Les archives de la douleur en yiddish“, im Druck (Anm. 2).

39 Siehe hierzu Sibylle Schmidt: Ethik und Episteme der Zeugenschaft, Konstanz 2015; A. Kalisky: „Jenseits der Typologien: die Vielschichtigkeit der Zeugenschaft“, in: Claudia Nickel und Alexandra Ortiz Wallner (Hg.): Zeugenschaft. Perspektiven auf ein kulturelles Phänomen, Heidelberg 2015, S. 193-211. 
gestalten, greift der Zeuge einerseits auf das Bezeugen zurück, das auf einer intendierten Referentialität seiner Aussage beruht. Andererseits greift er aber auch auf einen Gestus des „Wahrsprechens“ zurück, in welchem das Subjekt sich darstellt und sich performativ an die Wahrheit seiner eigenen Aussagen bindet. ${ }^{40}$ Das literarische und zugleich testimoniale Schreiben ist womöglich mit der Lyrik der Inbegriff des „Wahrsprechens“, insofern es, um die Worte Meschonnics zu verwenden, einen Diskurs definiert, der „vollkommen in den Zustand der Subjektivität versetzt ist“, in dem das „ich“ der Systematizität eines Diskurses verliehen wird. ${ }^{41}$ Da das testimoniale ,Er-zeugnis` selbst die Widersprüche, die es bestimmen, sowie die Übermittlungsmodi, die es anstrebt, definiert, ist es eine durch und durch reflexive Literatur, die wiederum zugleich ihre Form, ihren Sinn, ihren Wert und die Lektüre, die sie erfordert, reflektiert. Dieses testimoniale ,Er-zeugnis' versucht über sein Schicksal als Aussage, Archiv, Beweis und Dokument hinauszuwachsen, aber bleibt zugleich an die faktische Wahrheit durch einen ethischen Pakt gebunden, der garantiert, dass die literarisierte Wirklichkeit weiterhin der Wahrheit der erlebten Erfahrung und der Realität entspricht - selbst wenn dieses Entsprechen nicht den Regeln der Referentialität folgt.

Diese besondere Form von Zeugenschaft, die ich hier als eine dialektische Form zwischen Kunstwerk und Zeugnis analysiert habe, erfordert daher eine Lektüre, die sich des hybriden Wesens, der grundlegenden ,Unreinheit' und der paradoxen Poetik des Textes bewusst ist. Jeder kulturwissenschaftliche und philologische Interpretationsversuch dieser von Überlebenden geschriebenen Texte sollte die Herausforderung, die durch diese ganz eigene Form der Weitergabe gestellt wird, annehmen und versuchen, sowohl das Archiv, den Beweis, das literarische Werk und die Klage als Bestandteil derselben anzuerkennen. In dieser Hinsicht lädt der Text von Abraham Levite dazu ein, die Verhältnisse zwischen Kunst und Zeugenschaft im Zeitalter der „Krise der Zeugenschaft“ neu zu denken. Denn Levite schlägt nicht etwa vor - wie es Shoshana Felman in Hinblick auf Shoah von Claude Lanzman suggeriert - die ,Kunst der Zeugenschaft' als Prozess einer Stimmenverleihung durch die Kunst und den Künstler an die zum Schweigen gebrachten Opfer zu definieren. Die Kunst, die hier definiert wird, ist vor allem schon die Kunst der Opfer, die Kunst der Zeugen selbst. Diese Kunst wird nicht, wie bei Shoshana Felman, in den Zeugenstand berufen, um der Krise des westlichen Gesetzes der Evidenz entgegenzuwirken und die Geschichte der Opfer zu gründen. ${ }^{42}$ Die Opfer sind es, welche die göttliche wie menschliche Gerichtsszene eigens heraufbeschwören und sie selbst gestalten, um ihre „Stimme des Weinens“ laut werden zu lassen.

40 Giorgio Agamben: Das Sakrament der Sprache, Berlin 2010, p. 73.

41 Henri Meschonnic: Critique du rythme, Paris 2009, S. 86.

42 An dieser Stelle sei auf Felmans grundlegende sowie eindrucksvolle Studie verwiesen: Shoshana Felman: The juridical Unconscious: Trials and Traumas in the Twentieth Century, Cambridge 2002 . 